\title{
Serum apolipoprotein A1: a predictor and prognostic biomarker in acute ischemic stroke
}

\author{
Mai A. Eldeeb ${ }^{1}$ Amr S. Zaki ${ }^{*}$, Samia Ashour ${ }^{2}$, Azza Abdel Nasser², Ahmed El Bassiouny ${ }^{2}$ and \\ Khaled O. Abdulghani ${ }^{3}$
}

\begin{abstract}
Background: The apolipoprotein A1 level is recognized as a better indicator of cardiovascular disease than other cholesterol measures.

Objectives: To assess the serum level of ApoA1 in acute stroke patients and correlate it with the degree of vessel stenosis, stroke severity, prognosis, and functional outcome.

Patients and methods: We prospectively included 60 patients with first-ever cerebrovascular ischemic stroke, and they were matched with 30 healthy individuals matched in age and sex. Patients' neurological status was assessed via National Institute of Health and Stroke Scale (NIHSS). A venous blood sample was taken within the first $24 \mathrm{~h}$ of stroke onset and assayed for ApoA1 level by Human ApoA1 ELISA kit.
\end{abstract}

Results: ApoA1 level could be used to discriminate between cases and controls at a level of $6.2 \mu \mathrm{g} / \mathrm{ml}$, with $94.9 \%$ sensitivity and $86.6 \%$ specificity. Furthermore, there is an inverse relationship between the level of ApoA 1 and the clinical outcome expressed by NIHSS score and their prognosis after 3 months. Finally, there is an inverse relationship between ApoA1 level and the degree of stenosis whether intracranial or extracranial.

Conclusion: ApoA1 level can be used as a predictor of ischemic stroke and as a prognostic tool for those patients with ischemic stroke.

Keywords: Apolipoprotein A1, Stroke, Intracranial stenosis

\section{Introduction}

Apolipoprotein A1 (ApoA1) is the major protein component of high-density lipoprotein (HDL) and plays an important role in transporting excess cholesterol from peripheral cells to the liver. Besides the atheroprotective effect, ApoA1 also has anti-inflammatory and antioxidant effects. Recently, decreased serum ApoA1 level has been reported to increase the risk of deep subcortical infarction, which often coexists with white matter lesions in the brain [1]. Furthermore, the Honolulu-Asia aging study has found an inverse relation between ApoA1 and dementia [2]. In contrast, apolipoprotein $B(A p o B)$ is the major protein component of low-density lipoprotein (LDL). The

\footnotetext{
* Correspondence: amrsaeed@med.asu.edu.eg

${ }^{2}$ Neurology Department, Faculty of Medicine, Ain Shams University, Cairo,

Egypt

Full list of author information is available at the end of the article
}

oxidation of $\mathrm{ApoB}$ creates multiple proinflammatory products and propagates atherogenesis within the arterial wall [3].

The aim of this study was to assess the serum level of ApoA1 in patients with acute ischemic stroke compared to controls; to assess the relation between the serum ApoA1 level and their clinical picture, mortality, and their prognosis after 3 months; and finally to assess the relation between ApoA1 level and the degree of vessel stenosis.

\section{Subjects and methods}

A randomized prospective single-center study carried out during a 7-month period from April 2015 and October 2015 at Ain Shams University Hospitals. Ninety subjects were included in this study, divided into two groups: the case group that included 60 patients presented with acute 
ischemic cerebrovascular stroke (within $24 \mathrm{~h}$ from the onset of the stroke) and the control group that included 30 healthy individuals matched in age and sex. We excluded from the study patients with severe stroke (NIHSS more than 20) and individuals who had been on statins, calcitriol, and medication containing excess estrogen and fibrates. We also excluded individuals with previous history of ischemic heart disease, chronic renal failure, nephritic syndrome, cholestasis, and Alzheimer's disease. The study was approved by our local ethical committee, and informed written consent was signed by all included subjects.

Patients' neurological status was assessed via National Institute of Health and Stroke Scale (NIHSS), which was done on admission and after 3 months, and was classified as follows: $1-4=$ mild stroke, $5-15=$ moderate stroke, and 16-20 = moderate to severe stroke [4]. Laboratory investigations included HBA1c, LDL, HDL, triglycerides, and total cholesterol. Radiological investigations were including carotid duplex (General Electric Healthcare: LOGIQ F6; Waukesha, WI, USA) to assess the extra-cranial carotid artery, magnetic resonant brain imaging and magnetic resonant angiography for intracranial circulation (Philips MR Ingenia Ambition 1.5 T; Best, Netherlands), and echocardiography using transthoracic echocardiography.

Serum apolipoprotein A1 level using blood samples was collected from all subjects, and after centrifugation at $3000 \times$ for $10 \mathrm{~min}$, serum was obtained and stored at $-70^{\circ} \mathrm{C}$. For the quantitative detection of ApoA1, the Human ApoA1 ELISA kit, which is provided by Assay pro LLC (number: EA5201-1), was used and the instructions were done according to the provided booklet. The values of serum ApoA1 from each patient were noted and compared with values from the healthy controls in the laboratory of Ain Shams University hospitals.

\section{Statistical analysis}

The collected data was revised, coded, tabulated, and introduced to a PC using Statistical Package for Social Science (SPSS 15.0.1 for Windows; SPSS Inc., Chicago, IL, 2001). For descriptive statistics, we used mean, standard deviation $( \pm S D)$, and range for parametric numerical data, while median and interquartile range for nonparametric numerical data and frequency and percentage of non-numerical data. For analytical statistics, we used student $t$ test to assess the statistical significance of the difference between two study group means, MannWhitney test ( $U$ test) to assess the statistical significance of the difference of a non-parametric variable between two study groups, and correlation analysis (using Pearson's method) to assess the strength of association between two quantitative variables.

\section{Results}

The 60 patients with acute ischemic cerebrovascular stroke included 36 males (60\%) and 24 females (40\%) and their age range from 28 to 88 years old with mean age of $60 \pm 12.19$. Diabetes mellitus was the most prevalent risk factor in 38 patients (63.3\%), hypertension in 34 patients (56.7\%), dyslipidemia in 30 patients (50\%), and smoking in 23 patients (38.3\%). Those patients have been followed up after 3 months to assess their prognosis, compliance on their medication, recurrence of stroke, and mortality. Two of the patients dropped out during the follow-up period. Among the other $58 \mathrm{pa}$ tients, 56 patients $(94.9 \%)$ were compliant on their medications, 13 of them (22.8\%) showed recurrence of stroke, and 7 of them (11.7\%) unfortunately died.

By using Student $t$ test, ApoA1 measured in sera of ischemic stroke patients ranged from 1.7 to $21 \mu \mathrm{g} / \mathrm{ml}$ with mean $4.25 \mu \mathrm{g} / \mathrm{ml}$, while in the control group, it ranged from 2.8 to $21 \mu \mathrm{g} / \mathrm{ml}$ with mean $16.82 \mu \mathrm{g} / \mathrm{ml}$, thus revealing a high statistical significance difference between cases and controls, as shown in Fig. $1(p=0.001)$. Moreover, using Mann-Whitney test, there was also a highly significant difference between cases and controls with higher median among controls $(21 \mu \mathrm{g} / \mathrm{ml})$ versus cases $(4 \mu \mathrm{g} / \mathrm{ml})$. ROC curve showed that ApoA1 level could be used to discriminate between cases and controls at a level of $6.2 \mu \mathrm{g} / \mathrm{ml}$, with $94.9 \%$ sensitivity and $86.6 \%$ specificity.

Among those 60 ischemic strokes, 36 ischemic strokes (60\%) were located anterior, while 24 ischemic strokes (40\%) were located posterior. ApoA1 level measured in anterior strokes ranged from 2.8 to $4.45 \mu \mathrm{g} / \mathrm{ml}$ with median $4.2 \mu \mathrm{g} / \mathrm{ml}$ while in posterior strokes ranged from 2.5 to $4.1 \mu \mathrm{g} / \mathrm{ml}$ with median $3 \mu \mathrm{g} / \mathrm{ml}$ and thus revealing a statistical significance difference between anterior and posterior stroke indicating that lower level of ApoA1 is associated with posterior stroke rather than anterior ones.

Serum ApoA1 level was measured in those patients and correlated with their clinical outcome. And it revealed that the mean value of ApoA1 in patients with mild stroke (NIHSS 1-4) was $4.5 \pm 3.5 \mu \mathrm{g} / \mathrm{ml}$, while it decreased to 4.3 $\pm 4.0 \mu \mathrm{g} / \mathrm{ml}$ in patients with moderate stroke (NIHSS 515), and it showed even more decrease to $3.8 \pm 1.2 \mu \mathrm{g} / \mathrm{ml}$ in patients with moderate to severe stroke (NIHSS 16-20). In addition, it was correlated with their prognosis after 3 months and it revealed that the serum ApoA1 level was high (mean $4.4 \pm 4.0 \mu \mathrm{g} / \mathrm{ml}$ ) in patients with mild stroke, while it decreased to $4.2 \pm 1.9 \mu \mathrm{g} / \mathrm{ml}$ in patients with moderate stroke, and it logically decreased to $3.8 \pm 1.2 \mu \mathrm{g} / \mathrm{ml}$ in patients with moderate to severe stroke (Table 1).

ApoA1 level was assessed in all those patients, and its mean value in patients showing recurrence of stroke was $3.65 \pm 0.87 \mu \mathrm{g} / \mathrm{ml}$; however, it was higher in patients showing no recurrence of stroke (mean $4.46 \pm 3.85 \mathrm{ug} / \mathrm{ml}$ ), 


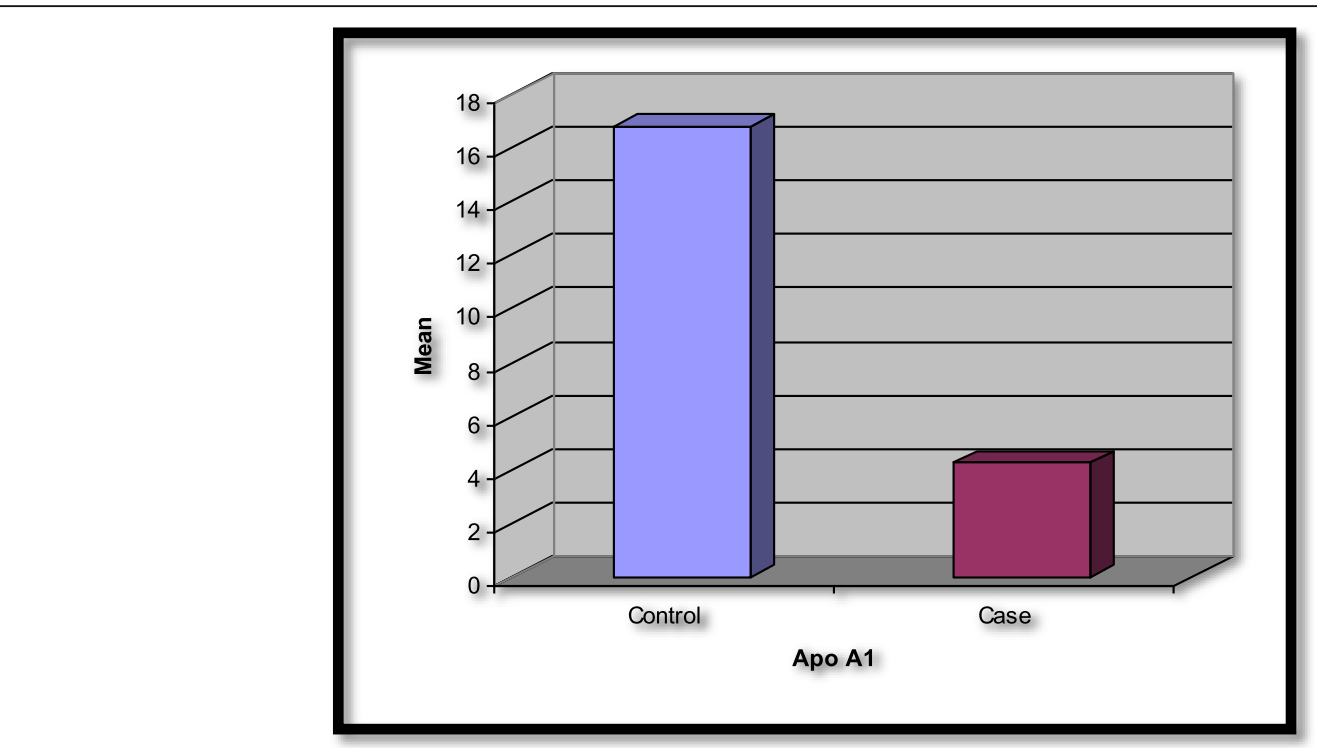

Fig. 1 Comparison between cases and controls as regards ApoA1 level using Student $t$ test

indicating that low level of ApoA1 acts as a predictor of recurrence of cerebrovascular stroke. However, these correlations did not reach statistical significance.

Furthermore, we classified patients into two groups based on their vascular imaging as follows. The intracranial atherosclerotic stenotic (ICAS) group is subdivided according to cerebral MRA into (a) normal (no stenosis), (b) mild (stenosis less than 50\%), (c) moderate (stenosis 50-69\%), (d) severe (stenosis 70-99\%), and (e) total occlusion. The other group was the extracranial atherosclerotic stenotic (ECAS) group which is subdivided into (a) normal, (b) diffuse atherosclerosis with no plaques, and (c) diffuse atherosclerosis with plaques. ApoA1 was assessed in each group and correlated with the degree of stenosis. ApoA1 levels were lowest in total occlusion in ICAS group and soft/calcific plaque in ECAS group, intermediate in stenosis in ICAS group and diffuse atherosclerosis in ECAS group, and highest in normal group in both ICAS and ECAS group. However, in

Table 1 Correlations between ApoA1 level and NIHSS at admission and after 3 months

\begin{tabular}{|c|c|c|c|c|}
\hline & & ApoA1 & & $P$ value \\
\hline & & Mean & $\pm S D$ & \\
\hline NIHSS baseline & Mild & 4.5 & 3.5 & 0.846 \\
\hline & Moderate & 4.3 & 4.0 & \\
\hline & Moderate to severe & 3.8 & 1.2 & \\
\hline NIHSS after 3 months & Mild & 4.4 & 4.0 & 0.888 \\
\hline & Moderate & 4.2 & 1.9 & \\
\hline & Moderate to severe & 3.8 & 1.2 & \\
\hline
\end{tabular}

ApoA1 apolipoprotein A1, NIHSS National Institute of Health and Stroke Scale general, ApoA1 level was lower in patients with ICAS than patients with ECAS (Table 2) (Fig. 2).

\section{Discussion}

The absence of a widely available and sensitive diagnostic and prognostic test for acute cerebral ischemia remains a significant limitation in the diagnosis, prognosis, and management of stroke. At most medical institutions, CT scans of the brain are performed as part of the initial evaluation of a patient with suspected stroke. The main advantage of this imaging modality is its widespread availability and sensitivity for hemorrhage. However, it is insensitive to early ischemic changes during acute cerebral ischemia and is usually of little value for establishing the diagnosis of acute stroke [5].

This raises the need to improve the prediction and diagnosis of stroke motivating the search for sensitive biomarkers. Research on the identification of novel

Table 2 Correlation between ApoA1 level and ICAS/ECAS

\begin{tabular}{lllll}
\hline & & \multicolumn{2}{l}{ ApoA1 } & P value \\
\cline { 3 - 4 } & & Mean & \pm SD & \\
\hline ICAS & Normal & 5.08 & 4.67 & 0.496 \\
& Stenosis less than 50\% (mild) & 3.88 & 0.81 & \\
& Stenosis 50-69\% (moderate) & 3.40 & 1.04 & \\
& Stenosis 70-99\% (severe) & 3.34 & 0.99 & \\
& Total occlusion & 3.23 & 1.24 & \\
\multirow{2}{*}{ ECAS } & Normal & 5.06 & 5.11 & 0.766 \\
& Diffuse atherosclerosis & 4.24 & 3.35 & \\
\hline
\end{tabular}

ApoA1 apolipoprotein A1, ICAS intracranial atherosclerotic stenotic, ECAS extracranial atherosclerotic stenotic 

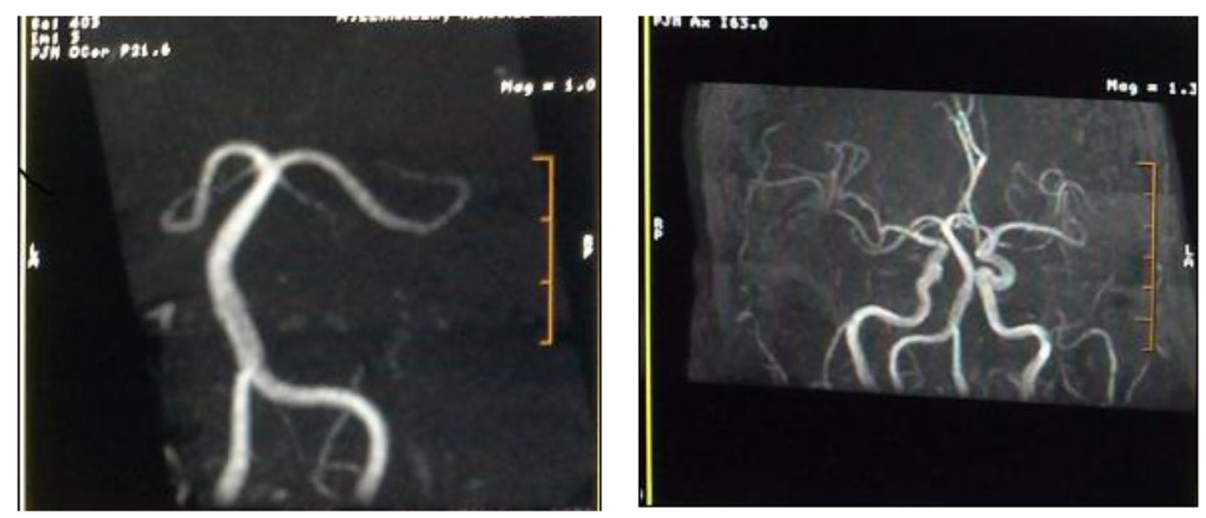

Fig. 2 MRA brain of patient presented with left insular, periventricular infarction, and diffuse atherosclerosis of intracranial vessels. NIHSS score was 5 on admission, 0 after 3 months, ApoA1 level was $5 \mu \mathrm{g} / \mathrm{ml}$

blood-borne markers that are associated with cerebral ischemia is continuing at a rapid pace, and it is likely that in the future, a biomarker or panel of biomarkers will play a role in facilitating the management of patients with cerebral ischemia. Such biomarkers may ultimately be used in a fashion similar to the use of troponin in the early diagnosis and management of patients with suspected myocardial ischemia or B-type natriuretic peptide in the diagnosis of congestive heart failure. Biomarkers may also be useful in the individualization of treatment decisions, determining etiology and providing prognostic information [6].

Ideally, a biomarker for diagnosing and monitoring prognosis of stroke should include at least the following characteristics. It should be brain specific. Because of obvious difficulties to obtain CSF samples in patients with acute stroke, detection in serum is highly desirable. It should appear very early, hours at the most, after the insult. Its peak level should reflect the extension of brain damage. It should possibly distinguish between stroke and stroke mimics, between transient and established stroke, and between hemorrhage and ischemia. Finally, it should be indicative of functional outcome [7].

That is why we decided to study apolipoprotein A1 in serum of stroke patients, because apart from differentiation between hemorrhage and ischemia, all the previous rules are applied to this specific marker.

Our main finding in this study is that low serum ApoA1 level is associated with an increased risk of ischemic cerebrovascular events. The cutoff value of ApoA1 level to discriminate between cases and controls was 6.2 $\mu \mathrm{g} / \mathrm{ml}$, with $94.9 \%$ sensitivity and $86.6 \%$ specificity. The mean value of ApoA1 in stroke patients versus healthy controls matched in age and sex revealed high statistical significance.

The previous finding is consistent with the results of a study by Kim and colleagues who reported that high serum ApoB level and low serum ApoA1 level are associated with increased risk of ischemic cerebrovascular events [8]. Also, it is consistent with the results of another study by Kostapanos and colleagues who reported that $\mathrm{ApoB} / \mathrm{ApoA} 1$ ratio may represent a predictor of a first-ever ischemic non-embolic stroke in elderly subjects which was evident after adjustment for other cardiovascular risk factors and confounders, including lipid parameters [9].

As an exploratory analysis, we further correlate the level of ApoA1 with the clinical outcome of the patient, recurrence of the stroke, and mortality. In AMORIS prospective study done by Walldius and colleagues, they showed that there is a strong direct relationship between the increasing values of $A p o B$, decreasing values of ApoA1, and the risk of fatal stroke [10]. Another study done by Bhatia and colleagues showed that there is an association between apolipoproteins and long-term risk of ischemic stroke in patients with previous transient ischemic attack [11]. Other recent studies strengthened the notion of the ApoB/ApoA1 ratio as a useful indicator of ischemic stroke risk in patients with preexisting atherothrombotic disease [12, 13]. These results are consistent with our findings.

In our study, we found that there is an inverse relationship between the level of ApoA1 and the clinical outcome expressed by NIHSS score. Also, cases who showed recurrence of stroke in the first 3 months after the incident of the first stroke had low ApoA1 level compared to those who did not show recurrence. Furthermore, cases that had low level of ApoA1 showed high mortality risk than cases of high ApoA1 level. However, all these correlations did not reach statistical significance. This could be explained by some reasons. First, we excluded severe stroke with NIHSS score $>20$, so we did not know the level of ApoA1 in such cases and we did not follow-up them. Secondly, due to ethical issues, we could not prevent the cases 
during follow-up from taking statin which increases the level of ApoA1 and reduces the risk of stroke. Finally, the small size of the sample can be an important factor.

Wallenfeldt and colleagues in their study approved that the ApoB/ApoA1 ratio was associated with metabolic syndrome and with the change in carotid artery intima medial thickness during 3 years of follow-up [14]. In addition, Park and colleagues in their study concluded that higher $\mathrm{ApoB} / \mathrm{ApoA} 1$ ratio is a predictor of ICAS rather than of ECAS and that ApoB/ApoA1 ratio might be a biomarker for ICAS in patients with stroke [15].

These findings were present in our study. The mean level of ApoA1 in patients with no intracranial atherosclerotic stenosis was $5.08 \pm 4.67 \mu \mathrm{g} / \mathrm{ml}$, and it decreased in patients with mild ICAS and decreased with increasing stenosis till reaching its lowest level in patients with severe stenosis or occlusion. Also, in patients with ECAS, it was $5.06 \pm 5.11 \mu \mathrm{g} / \mathrm{ml}$ in patients with no stenosis and it further decreased with the appearance of plaques. But in general, ApoA1 level was lower in patients with ICAS than patients with ECAS. However, all these correlations did not reach statistical significance, and this could be explained by the small size of the sample.

\section{Conclusion}

ApoA1 level can be used as a predictor of ischemic stroke and as a prognostic tool for those patients with ischemic stroke. Further studies are needed to measure the ApoA1 level in the patients during follow-up. In addition, multi-center with a greater scale of patients is recommended.

\section{Abbreviations}

ApoA1: Apolipoprotein A1; ApoB: Apolipoprotein B; ECAS: Extracranial atherosclerotic stenotic; HBA1c: Hemoglobin A1c; HDL: High-density lipoprotein; ICAS: Intracranial atherosclerotic stenotic; LDL: Low-density lipoprotein; NIHSS: National Institute of Health and Stroke Scale; ROC: Receiver operating characteristic

\section{Acknowledgements}

Not applicable

\section{Authors' contributions}

$S A, A A$, and $A B$ made substantial contributions to conception and design of the study. $A Z$ performed the acquisition of data. $M E, A Z, A A$, and $A B$ participated in the analysis and interpretation of data. AZ and KA have been involved in drafting the manuscript or revising it critically for important intellectual content. AA has given final approval of the version to be published. All authors agreed to be accountable for all aspects of the work in ensuring that questions related to the accuracy or integrity of any part of the work are appropriately investigated and resolved. All authors read and approved the final manuscript.

\section{Funding}

None

\section{Availability of data and materials}

The datasets generated and analyzed during the current study are not publicly available due to institutional limitations, yet they are available from the corresponding author on reasonable request.

\section{Ethics approval and consent to participate}

This study was approved by the ethical committee of Ain Shams University School of Medicine on the 5th of April 2015. All subjects provided informed written consent prior to the participating in any study-related activities.

\section{Consent for publication}

Not applicable

\section{Competing interests}

The authors declare that they have no competing interests.

\section{Author details}

${ }^{1}$ Internal Medicine Department, Faculty of Medicine, Ain Shams University, Cairo, Egypt. ${ }^{2}$ Neurology Department, Faculty of Medicine, Ain Shams University, Cairo, Egypt. ${ }^{3}$ Neurology Department, Helwan University School of Medicine, Cairo, Egypt.

Received: 28 October 2019 Accepted: 2 December 2019

Published online: 31 December 2019

\section{References}

1. Park JH, Hong KS, Lee J, Kim YJ, Song P. Deep subcortical infarct burden in relation to apolipoprotein $\mathrm{B} / \mathrm{Al}$ ratio in patients with intracranial atherosclerotic stenosis. Eur J Neurol. 2013;20(4):671-80.

2. Saczynski JS, White L, Peila RL, Rodriguez BL, Launer $L$ J. The relation between apolipoprotein A-I and dementia: the Honolulu-Asia aging study. Am J Epidemiol. 2007;165(9):985-92.

3. Sniderman AD, Faraj M. Apolipoprotein B, Apolipoprotein A-I, insulin resistance and the metabolic syndrome. Curr Opin Lipidol. 2007;18(6):633-7.

4. Spilker J, Kongable G, Barch C, Braimah J, Brattina P, Daley S, et al. Using the $\mathrm{NIH}$ Stroke Scale to assess stroke patients. The NINDS rt-PA Stroke Study Group. J Neurosci Nurs. 1997;29(6):384-92.

5. Fiebach JB, Schellinger PD, Jansen $O$, Meyer $M$, Wilde $P$, Bender J, et al. CT and diffusion-weighted MR imaging in randomized order: diffusionweighted imaging results in higher accuracy and lower interrater variability in the diagnosis of hyperacute ischemic stroke. Stroke. 2002;33(9):2206-10.

6. Kernagis DN, Laskowitz DT. Evolving role of biomarkers in acute cerebrovascular disease. Ann Neurol. 2012;71(3):289-303.

7. Zimmermann-Ivol CG, Burkhard PR, Le Floch-Rohr J, Allard L, Hochstrasser DF, Sanchez JC. Fatty acid binding protein as a serum marker for the early diagnosis of stroke: a pilot study. Mol Cell Proteomics. 2004;3(1):66-72.

8. Kim BS, Jung HS, Bang OY, Chung CS, Lee KH, Kim GM. Elevated serum lipoprotein(A) as a potential predictor for combined intracranial and extracranial artery stenosis in patients with ischemic stroke. Atherosclerosis. 2010;212:682-8.

9. Kostapanos MS, Christogiannis LG, Bika E, Bairaktari ET, Goudevenos JA, Elisaf MS, et al. Apolipoprotein B-to-A1 ratio as a predictor of acute ischemic nonembolic stroke in elderly subjects. J Stroke Cerebrovasc Dis. 2010;19(6):497-502.

10. Walldius G, Aastveit AH, Junger I. Stroke mortality and the apoB/apo A-I ratio: results of the AMORIS prospective study. J Intern Med. 2006;259(3):259-66.

11. Bhatia M, Howard SC, Clark TG, Neale R, Qizilbash N, Murphy MF, et al. Apolipoproteins as predictors of ischemic stroke in patients with a previous transient ischemic attack. Cerebrovascular Dis. 2006;21(5-6):323-8.

12. Wang A, Xu J, Chen G, Wang D, Johnston SC, Meng X, et al. Oxidized lowdensity lipoprotein predicts recurrent stroke in patients with minor stroke or TIA. Neurology. 2018;91:e947-55.

13. Sun $Y$, Hou XH, Wang DD, Ma YH, Tan CC, Sun FR, et al. Apolipoprotein B/Al ratio as an independent risk factor for intracranial atherosclerotic stenosis. Aging (Albany NY). 2019;11(17):6851-62.

14. Wallenfeldt K, Bokemark L, Wikstrand J, Hulthe J, Fagerberg B. Apolipoprotein B/apolipoprotein A I in relation to the metabolic syndrome and change in carotid artery intima media thickness during 3 years in middle-aged men. Stroke. 2004;35(10):2248-52.

15. Park JH, Hong KS, Lee EJ, Lee J, Kim DE. High levels of apolipoprotein B/AI ratio are associated with intracranial atherosclerotic stenosis. Stroke. 2011; 42(11):3040-6

\section{Publisher's Note}

Springer Nature remains neutral with regard to jurisdictional claims in published maps and institutional affiliations. 